Media- und Marketingstrategien in digitalen Zeiten 
Anja Schüür-Langkau (Hrsg.)

\section{Media- und \\ Marketingstrategien in digitalen Zeiten}

Trendinterviews mit Branchen-Experten aus Wissenschaft und Praxis

Springer Gabler 
Herausgeber

Anja Schüür-Langkau

Springer Fachmedien Wiesbaden GmbH

Wiesbaden, Deutschland

Die Deutsche Nationalbibliothek verzeichnet diese Publikation in der Deutschen Nationalbibliografie; detaillierte bibliografische Daten sind im Internet über http://dnb.d-nb.de abrufbar.

Springer Gabler

(C) Springer Fachmedien Wiesbaden 2012

Das Werk einschließlich aller seiner Teile ist urheberrechtlich geschützt. Jede Verwertung, die nicht ausdrücklich vom Urheberrechtsgesetz zugelassen ist, bedarf der vorherigen Zustimmung des Verlags. Das gilt insbesondere für Vervielfältigungen, Bearbeitungen, Übersetzungen, Mikroverfilmungen und die Einspeicherung und Verarbeitung in elektronischen Systemen.

Die Wiedergabe von Gebrauchsnamen, Handelsnamen, Warenbezeichnungen usw. in diesem Werk berechtigt auch ohne besondere Kennzeichnung nicht zu der Annahme, dass solche Namen im Sinne der Warenzeichen- und Markenschutz-Gesetzgebung als frei zu betrachten wären und daher von jedermann benutzt werden dürften.

Lektorat: Angela Pfeiffer

Gedruckt auf säurefreiem und chlorfrei gebleichtem Papier

Springer Gabler ist eine Marke von Springer DE. Springer DE ist Teil der Fachverlagsgruppe Springer Science+Business Media.

www.springer-gabler.de 


\section{Vorwort}

Die Spielregeln des Werbemarktes haben sich verändert. Alle Akteure sind betroffen - ob Konsumenten, Werbungtreibende, Agenturen oder Medien. Durch den technischen Fortschritt und eine sich verändernde Mediennutzung entwickelt sich eine Vielzahl neuer Kanäle, die das Marktgefüge gravierend beeinflussen. Werbekunden, Agenturen, Marktforscher, Medien und auch die Wissenschaft müssen daher ihre Strategien im Marketing neu überdenken.

Im vorliegenden Buch analysieren Branchen-Experten in ausführlichen Interviews mit dem Fachmagazin media spectrum die aktuelle Situation und zeigen unterschiedliche Sichtweisen, Herangehensweisen und Lösungsansätze auf. Ausgewählte Best-Practice-Beispiele aus verschiedenen Branchen ergänzen den Marktüberblick.

Vor allem die werbungtreibenden Unternehmen müssen sich zunehmend mit der Frage auseinandersetzen, wie sie mit ihren Kampagnen auch ihre Ziele erreichen und einen messbaren Return on Investment erwirtschaften. Vor dem Hintergrund wachsenden Einflusses des Controllings wird es für viele Marketingverantwortliche jedoch immer schwieriger, erfolgreiche Kommunikation zu betreiben.

Marktforschungsunternehmen erweitern angesichts der neuen Konsumenten-Souveränität ihr Methodenspektrum. Konzepte wie Open Innovation und Co-Creation, Crowdsourcing und Mass Customization halten Einzug in die Marktforschungspraxis. Für die Branche erwachsen daraus neue Chancen. Doch der Wettbewerb wird härter, und die Marktforschung muss sich neu positionieren.

Die Aufgabe der Mediaagenturen ist es, die Werbekunden strategisch bei der Planung ihrer Kommunikationsmaßnahmen zu beraten und deren Umsetzung erfolgreich zu orchestrieren. Dabei wird die Steuerung einer Kampagne über mehrere Marketingkanäle hinweg vor dem Hintergrund fragmentierter Märkte und zunehmender Medienkonvergenz immer schwieriger. Hinzu kommt der seit Jahren anhaltende Renditedruck, der Mediaagenturen zwingt, den Konditionendruck auf die Medien zu erhöhen.

Die Medienunternehmen hingegen stehen zwischen den Fronten. Zum einen müssen sie auf Grund stagnierender und zum Teil sinkender Reichweiten und veränderter Nutzungsstrukturen neue Wege finden, um Leser, Zuschauer und Hörer zu binden und ihre Angebote mit den digitalen Medien zu vernetzen. Zum anderen stehen sie in der Pflicht, ihre Leistungen und Wirkungen detaillierter nachzuweisen und müssen sich dennoch dem Rabattdruck der Agenturen und Werbekunden beugen.

Die Marketing-Wissenschaft setzt sich ebenfalls intensiv mit den neuen Entwicklungen auseinander. Doch der Transfer in die Praxis lässt oft zu wünschen übrig. Die Diskussion, wie Wissenschaft und Praxis künftig stärker voneinander profitieren können, wird derzeit intensiv geführt.

Wiesbaden

Anja Schüür-Langkau 


\section{Inhalt}

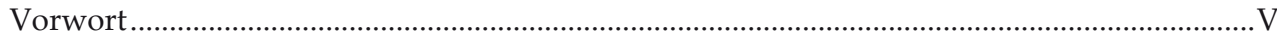

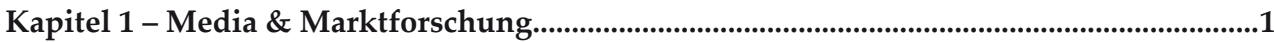

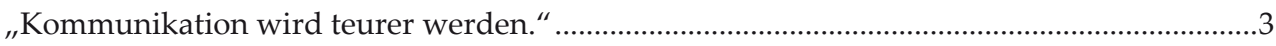

Interview mit Thomas Koch, tk-one, Düsseldorf

„Wir müssen den Paradigmenwechsel in jeder Form begleiten. “ ..............................................9

Interview mit Jens-Uwe Steffens, Pilot, Hamburg

Methoden aus einer gestrigen Welt

Interview mit Thomas Strerath, Ogilvy \& Mather Deutschland GmbH

Werbetreibende oft sich selbst überlassen.

Interview mit Thorsten Müller, Reckitt Benckiser

„Die klassische Media-Logik löst sich auf.“ .21

Interview mit Prof. Dr. Wolfgang Schweiger, TU Ilmenau

Mobilfunk ist ein globaler Megatrend.

Interview mit Christoph Keese, Axel Springer

„Es gibt keinen größeren Erlöshebel als Paid Content.“

Interview mit Gregor Waller, Frenemies Consulting

„Qualität ist das Leitmotiv unserer Strategie."

Interview mit Tobias Trevisan, Frankfurter Allgemeine Zeitung

Crossmediale Reichweite soll Zeitungsvermarktung unterstützen.

Interview mit Markus Ruppe, ZMG Zeitungs Marketing Gesellschaft, Frankfurt

„Die Research-Zyklen sind dramatisch schneller geworden."

Interview mit Ralf Ganzenmüller, Ipsos Deutschland

Multitasken macht dumm

Interview mit Professor Dr. Dr. Manfred Spitzer, Universitätsklinik Ulm

Kapitel 2 - Marketing \& Markenführung .47

„Learn to drive before you fly." .49

Interview mit Dr. Jesko Perrey, McKinsey \& Company, Düsseldorf 
Die Markenarchitektur wird immer wichtiger 54

Interview mit Prof. Dr. Torsten Tomczak, Universität St. Gallen

„So viel Standardisierung wie möglich - so viel Differenzierung wie nötig“ 58

Interview mit Prof. Dr. Dr. h. c. mult.Heribert Meffert, Universität Münster

Social-Media-Maßnahmen stützen das Image von Marken .

Interview mit Prof. Dr. Manfred Bruhn, Universität Basel

Social Media revolutioniert das Marketing

Interview mit Prof. Dr. Tobias Langner, Bergische Universität Wuppertal

"Vollmundige Slogans sind online wertlos."

Interview mit Ossi Urchs, Strategieberater

„Die strategische Überlegung muss immer am Anfang stehen."

Interview mit Prof. Dieter Kempf, Bitkom und DATEV

Echte Verknüpfung der Vertriebskanäle ist die hohe Kunst der nächsten Jahre

Interview mit Prof. Dr. Dirk Morschett, Universität Fribourg

Rolle des Pricings wird immer noch unterschätzt

Interview mit Dr. Rainer Meckes, Simon-Kucher E Partners

Markenführung ist auch Mitarbeiterführung.

Interview mit Prof. Dr. Holger Rust, Universität Hannover

Raus aus der Einbahnstraßen-Kommunikation

Interview mit Hubert Sichler, Serviceplan Health \& Life

Kapitel 3 - Ausgewählte Best-Practice-Beispiele

„Social Media erzeugen eine gnadenlose und gute Transparenz."

Interview mit Thomas Voigt, Otto Group

„Nur einem beständigen Unternehmen schenkt man Vertrauen.“

Interview mit Orazio Costadura, Audi AG

„Unsere Kernziele sind Imageverbesserung und echte Transparenz." .

Interview mit Carmen Borsche, Nestlé Deutschland AG

Der Markenkern steuert die Kommunikation

Interview mit Anders-Sundt Jensen, Mercedes-Benz Cars

„Wir dürfen Effizienz nicht nur quantitativ bewerten."

Interview mit Margret Dreyer, Postbank 